\title{
Acute acalculous cholecystitis in a 17-year-old girl with Epstein-Barr virus infection
}

\author{
Agnieszka Pawłowska-Kamieniak ${ }^{1}$, Agnieszka Mroczkowska-Juchkiewicz ${ }^{1}$, Dorota Gołyska ${ }^{1}$, \\ Katarzyna Kominek², Elżbieta Pac-Kozuchowska ${ }^{2}$ \\ Department of Paediatric, Medical University of Lublin, Lublin, Poland
}

Prz Gastroenterol 2015; 10 (1): 54-56 DOI: $10.5114 / p g .2015 .48998$

Key words: acalculous cholecystitis, Epstein-Barr virus, children.

Address for correspondence: Agnieszka Mroczkowska-Juchkiewicz MD, PhD, Department of Paediatric, Medical University of Lublin, 2 Chodźki St, 20-093 Lublin, Poland, phone: +48 8171854 20, e-mail: aga juchkiewicz@poczta.onet.pl

\begin{abstract}
Acute cholecystitis is most frequently concomitant with cholelithiasis, whereas acute acalculous cholecystitis is usually of an infectious aetiology. Among the aetiological factors, Epstein-Barr virus (EBV) infection is also mentioned. The case of a 17-yearold girl is described, hospitalised in the Children's Clinical Hospital, Paediatric Clinic, at the Medical University in Lublin, due to fever, upper abdomen pain lasting for a week, and nausea for several days. Based on the diagnostic - laboratory tests performed and ultrasonographic examination, acute acalculous cholecystitis was diagnosed, taking course with elevated aminotransferase activity and features of cholestasis. Serological tests confirmed an acute infection with Epstein-Barr virus. After 2 weeks of hospitalisation, the patient, receiving conservative treatment, was discharged home in good condition. A follow-up examination performed 2 weeks later did not show deviation from normal.
\end{abstract}

\section{Introduction}

Acute cholecystitis is most frequently concomitant with cholelithiasis, whereas acute acalculous cholecystitis (ACC) is usually of an infectious aetiology. It occurs in the course of bacterial diseases, both systemic and in the case of purulent processes that take place in the vicinity of the gall bladder, and sometimes as a complication of surgical procedures performed on the bile duct. Among the bacteria cultured from bile the following are mentioned: streptococci, staphylococci, anaerobic bacteria, and Gram-negative [1-3]. Epstein- Barr virus is known to be one of the factors inducing viral hepatitis. $A$ rare consequence of EBV infection is acute ACC [1-4].

\section{Case report}

A case of a 17-year-old girl is described, hospitalised in the Children's Clinical Hospital, Paediatric Clinic, at the Medical University in Lublin, due to fever, upper abdomen pain lasting for a week, and nausea for several days. At admission, the patient's body temperature was high, she complained of pain in the right hypochondrium, and nausea. In the physical examination, inflammatory changes were diagnosed in the adenoid glands, pain on palpation in the middle and right epigastrium. Ultrasound scan of the abdominal cavity showed the acalculous gallbladder with an irregular wall thickened to $7 \mathrm{~mm}$, and single lymph nodes in the hepatic hilum. In laboratory tests, elevated markers of the inflammatory state were observed (moderate C-reactive protein (CRP) and erythrocyte sedimentation rate (ESR), high leucocytosis with relative lymphocytosis), elevated aminotransferase activity (alanine aminotransferase (ALT) - $268 \mathrm{U} / \mathrm{l}$, aspartate aminotransferase (AST) - $230 \mathrm{U} / \mathrm{l}$ ), and features of cholestasis ( $\gamma$-glutamyl transferase (GGTP) $135 \mathrm{U} / \mathrm{l}$, alkaline phosphatase (ALP) - $311 \mathrm{U} /$, bilirubin $2.2 \mathrm{mg} / \mathrm{dl}$ ). On the day of admission, the latex test was performed for infectious mononucleosis, and a positive result was obtained. Viral infection with hepatitis $B$ virus (HBV), hepatitis $C$ virus ( $\mathrm{HCV}$ ), and cytomegalovirus (CMV) was excluded, and an acute Epstein-Barr virus (EBV) infection was serologically confirmed (immunoglobulin M (IgM) - $168 \mathrm{AU} / \mathrm{ml}$, immunoglobulin G (IgG) $-21.4 \mathrm{AU} / \mathrm{ml})$.

Based on the results of laboratory tests and ultrasonographic examination performed, an acute ACC was diagnosed, taking course with an elevated aminotransferase activity and features of cholestasis. 
Antibiotic therapy, ursodeoxycholic acid (UDCA), analgesics, and relaxants were implemented into the treatment. After 2 weeks of hospitalisation, the patient was discharged home in good condition. Follow-up laboratory tests performed after 2 weeks did not show any deviations from the normal.

\section{Discussion}

In adult patients, an acute ACC constitutes $5-10 \%$ of all cases of cholecystitis; however, it occurs considerably less frequently among children [2, 4, 5]. The aetiology of this inflammation is generally bacterial, and very rarely viral $[1-4,6]$.

The diagnosis of acute ACC is made based on clinical symptoms, laboratory tests, and ultrasonographic image.

The ACC usually manifests itself as pain in the right epigastric region, occurring as a general rule from $1-2 \mathrm{~h}$ after a meal. The pain is not colicky in character, quickly achieves maximum intensity, and may last a relatively long time (more than $6 \mathrm{~h}$ ), radiating to the right shoulder blade or back. It is frequently accompanied by nausea or vomiting and moderate fever $[4,5]$. Signs and symptoms of acute cholecystitis are similar to other causes of acute abdominal pain such as pancreatitis or gastritis [7]. In physical examination, pain and abdominal guarding is diagnosed in the right hypochondrium, with a positive Murphy's sign [1-3].

Based on laboratory tests performed in a patient with acute ACC, elevated inflammatory parameters are usually observed, sometimes with elevated aminotransferase and GGTP activity, and increased levels of bilirubin $[5,6]$.

The ultrasonography (USG) is a basic imaging examination in the diagnostics of acute cholecystitis, its efficiency being assessed at approximately $90 \%$. The diagnosis is made based on the thickening of the gallbladder wall and distention of its lumen [1, 3, 5].

The treatment of uncomplicated, acute ACC is conservative and symptomatic. It consists of the application, according to needs, of analgesics, antiemetics, antibiotic therapy, and UDCA [3].

Epstein-Barr virus infection in immunocompetent individuals may be asymptomatic or it may manifest itself by high fever, inflammatory changes in the throat, and enlarged peripheral lymph nodes. The EBV can also be among the factors causing viral hepatitis [1-4]. A rare symptom of Epstein-Barr virus infection is an acute ACC [1-4]. Cholestasis may be due to bile duct inflammation or enlarged lymphatic tissue in the course of EBV infection [3].

There are few literature reports confirming a viral aetiology, especially EBV, as the cause of ACC.

Sirmatel et al. described 5 cases of acute acalculous cholecystitis with a typical medical history and clinical symptoms. In the course of the diagnostic procedure, in association with elevated titres of aminotransferase with cholestasis features, all patients had tests performed for viral hepatitis; positive titres were obtained in IgM class to EBV infection [3]. Attilakos et al. reported 2 cases of ACC in the course of EBV infection in 2 children with Gilbert's syndrome [1].

laria et al. presented the case of an 18-year-old girl with acute acalculous cholecystitis due to EBV infection [2]. Prassouli et al. described a case of a 13-year-old girl with EBV infection and concomitant cholestatic hepatitis and AAC [4].

Other viral aetiology of AAC was dewscribed in the literature Herek et al. The investigators presented a case of a 9-year-old boy with acute acalculous cholecystis in the course of hepatitis A infection [6].

Acute ACC in the course of cystic fibrosis was presented by Shihabuddin and Sivitz [5]. Symptoms and signs of AAC, similar to other causes of acute abdominal pain localised in the right-upper quadrant of the abdomen, make diagnosis difficult [5, 7].

The presented case of a patient hospitalised in the Paediatric Clinic satisfied all diagnostic criteria for ACC. Medical history, physical examination, and results of auxiliary examinations suggested the necessity to take into consideration, in the differential diagnostics, cholestasis and inflammation of the gallbladder, as well as a viral infection, including EBV infection.

\section{Conclusions}

Among the causes of acute ACC Epstein-Barr virus infection should be taken into consideration. Acute ACC of EBV aetiology is a self-limiting process, treated conservatively.

\section{Conflict of interest}

The authors declare no conflict of interest.

\section{References}

1. Attilakos A, Prassouli A, Hadjigeorgiou G, et al. Acute acalculous cholecystitis in children with Epstein-Barr virus infection: a role for Gilbert's syndrome? Int J Infect Dis 2009; 13: 161-4.

2. Iaria C, Arena L, Di Maio G, et al. Acute acalculous cholecystitis during the course of primary Epstein-Barr virus infection: a new case a review of the literature. Int J Infect Dis 2008; 12: 391-5.

3. Sirmatel O, Sirmatel F, Eris FN, et al. The cases of cholestatic hepatis in the course of atypical Epstein-Barr virus infection. Res J Med Sci 2010; 4: 136-41.

4. Prassouli A, Panagiotou J, Vakaki M, et al. Acute acalculous cholecytitis as the initial presentation of primary Epstein-Barr virus infection. J Pediatr Surg 2007; 42: 11-3.

5. Shihabuddin B, Sivitz A. Acute acalculous cholecytitis in a 10-year-old girl with cystic fibrosis. Pediatr Emerg Care 2013; 29: 117-21. 
6. Herek O, Cördük N, Herek D, et al. Acute acalculous cholecystitis due to hepatitis $A$ infection in a child: a rare cause of acute abdomen. Ann Afr Med 2011; 10: 193-5.

7. Oracz G, Kierkuś J, Socha J, Ryżko J. The clinical course of chronic pancreatitis associated with lipid disturbances in children [Polish]. Prz Gastroenterol 2012; 7: 286-90.

Received: 23.11.2012

Accepted: 12.02.2013 